Please do not remove this page

RMIT

UNIVERSITY

\title{
Model based testing for agent systems
}

Zhang, Zhiyong; Thangarajah, John; Padgham, Lin

https://researchrepository.rmit.edu.au/esploro/outputs/9921861610101341/filesAndLinks?institution=61RMIT_INST\&index=null

Zhang, Z., Thangarajah, J., \& Padgham, L. (2007). Model based testing for agent systems. Software and Data Technologies. https://doi.org/10.1007/978-3-540-88655-6_30

Document Version: Accepted Manuscript

Published Version: https://doi.org/10.1007/978-3-540-88655-6_30

Repository homepage: https://researchrepository.rmit.edu.au

(C) 2007 Springer Berlin Heidelberg

Downloaded On 2023/04/26 09:11:27 +1000

Please do not remove this page 


\title{
AUTOMATED UNIT TESTING FOR AGENT SYSTEMS
}

\author{
Zhiyong Zhang, John Thangarajah, Lin Padgham \\ School of Computer Science, RMIT, Melbourne, Australia \\ $\{$ zhzhang,johthan,linpa\}@cs.rmit.edu.au
}

Citation: Zhang, Z, Thangarajah, J and Padgham, L 2007, 'Model based testing for agent

Keywords: Agent-Oriented Software Engineering, Multi-Agent Systems, Unit Testing

Abstract: Although agent technology is gaining world wide popularity, a hindrance to its uptake is the lack of proper testing mechanisms for agent based systems. While many traditional software testing methods can be generalized to agent systems, there are many aspects that are different and which require an understanding of the underlying agent paradigm. In this paper we present certain aspects of a testing framework that we have developed for agent based systems. The testing framework is a model based approach using the design models of the Prometheus agent development methodology. In this paper we focus on unit testing and identify the appropriate units, present mechanisms for generating suitable test cases and for determining the order in which the units are to be tested, present a brief overview of the unit testing process and an example. Although we use the design artefacts from Prometheus the approach is suitable for any plan and event based agent system.

\section{Introduction}

Agent systems are increasingly popular for building complex applications that operate in dynamic domains, often distributed over multiple sites. While the dream of theory based verification is appealing, the reality is that these systems are reliant on traditional software testing to ensure that they function as intended. While many principles can be generalised from testing of object oriented systems (Binder, 1999), there are also aspects which are clearly different and that require knowledge of the underlying agent paradigm.

For example in many agent systems paradigms (including BDI - Belief, Desire, Intention [(Rao and Georgeff, 1995)]) there is a concept of an event which triggers selection of one of some number of identified plans, depending on the situation. If one of these plans is actually never used, then this is likely to indicate an error. The concepts of event and plan, and the relationships between them are part of typical agent designs, and can thus be used for model based testing of agent systems. Effective testing of an agent system needs to take account of these kinds of relationships.

In this paper, we describe some of the aspects of a framework we have developed to automatically gen- erate unit test cases for an agent system, based on the design models. The testing framework includes components that generate the order in which the units are to be tested, generate inputs for creating test cases, automate the test case execution, augment the system code to enable the testing to be performed, and a test agent that activates the testing process, gathers the results and generates a report that is easily understood.

We base our approach on the notion of model based testing [(Apfelbaum and Doyle, 1997; El-Far and Whittaker, 2001)] which proposes that testing be in some way based on design models of the system. There are a number of agent system development methodologies, such as Tropos (Bresciani et al., 2004), Prometheus (Padgham and Winikoff, 2004), MASE (Deloach, 2001) and others, which have well developed structured models that are potentially suitable as a basis for model based testing. In our work we use the Prometheus models. The models that are developed during design provide information against which the implemented system can be tested, and also provide an indication of the kind of faults that one might discover as part of a testing process.

There has been some work by others on testing agent systems in recent years. However, they have 
either focused on testing for the properties of abstract BDI-agents (Zheng and Alagar, 2005), or performed black box testing of the system (Seo et al., 2004).

In this paper, we focus on unit testing the components of a single agent. Unlike more traditional software systems, such as those based on Object-Oriented principles, where the base units are classes that are called via method invocation, the units in agent systems are more complex in the way they are called and are executed. For instance, plans are triggered by events, an event may be handled by more than one plan, plans may generate events that trigger other plans either in sequence or in parallel and so on. A testing framework for agent based systems must take these details into consideration in identifying the appropriate units and developing appropriate test cases.

In the sections ahead, we first identify what the natural units for an agent based system are, and how we use the model to determine the various test cases and their expected outcomes. We then provide an overview of the testing process and provide details on the reasoning that is done regarding dependencies between units, the necessary ordering of test cases, and the way in which inputs are generated for the various test cases. We provide a brief example from the evaluation with a case study, and then conclude with a discussion that identifies related and future work.

\section{Test Units}

The type of testing that we perform is faultdirected testing, where we intend to reveal faults in the implementation through failures (Binder, 1999, p.65). This is in contrast to conformance-based testing, which tests whether the system meets the business requirements. ${ }^{1}$

In order to perform fault-directed testing we require knowledge about the failures that can occur within the design paradigm (often called the fault model). In this section, we identify the units to be tested and identify possible points of failure for each unit that are independent of the implementation. We begin by examining the Prometheus design artefacts to identify suitable units for testing. Figure 1 outlines the components of an agent within the Prometheus methodology. ${ }^{2}$ An agent may consist of plans, events and belief-sets, some of which may be encapsulated into capabilities. Percepts and incoming messages are

\footnotetext{
${ }^{1}$ We expect to eventually also add conformance based testing, using use cases and other artefacts from the models developed at the requirements analysis stage, rather than the detailed design models being used here.

${ }^{2}$ Other agent oriented methodologies use similar constructs.
}

inputs to the agent, while actions and outgoing messages are outputs from the agent.

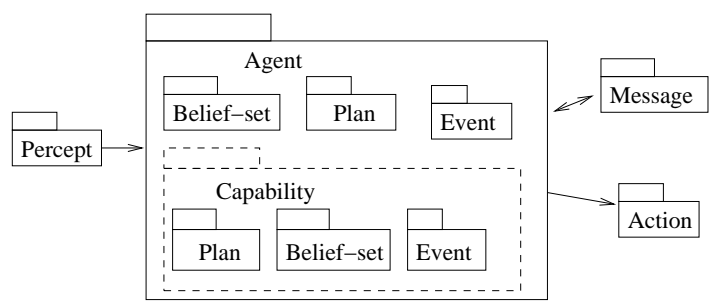

Figure 1: Agent Component Hierarchy in Prometheus

Beliefsets are essentially the agent's knowledge about the environment and therefore constitute the situations in which testing must be done. The basic units of testing then are the plans and the events. Percepts and messages are also treated as events in agent development tools like JACK (Busetta et al., 1999) and similar systems, and we also use this same generalisation.

We now discuss informally appropriate fault models for testing plans and events.

\subsection{Testing Plans}

A plan in its simplest form consists of a triggering event, a context condition, which determines the applicability of the plan with respect to the agent's beliefs about the current state of the world, and a plan body which outlines a sequence of steps. These steps may be subtasks, activated by posting events that are handled by the agent itself or external message events, which will be handled by another agent.

When we consider a plan as a single unit we test for the following aspects:

1. Does the plan get triggered by the event that it is supposed to handle? If it does not, then there could be two possible reasons. The first is that some other plan always handles it, and the other is that there could be an inconsistency between the design and code and no plan actually handles that particular event ${ }^{3}$.

2 . Is the context condition valid? The context condition for a plan is optional. The absence of a context condition denotes that the plan is always applicable. However, if the designer includes a context condition, then it should evaluate to true in at least one situation and not in all.

3. Does the plan post the events that it should? Events are posted from a plan to initiate sub-tasks

\footnotetext{
${ }^{3}$ Here we can only check if the design matches the code, and can not check, for example, if the plan handling a particular event is correct or sensible.
} 
or send messages. If some expected events are never posted, we need to identify them as this may be an error.

4. Does the plan complete? While it is difficult to determine whether a plan completes successfully or not, we can at least determine whether the plan executed to completion. If the plan does not complete then there is an error. ${ }^{4}$ In implementation systems like JACK(Busetta et al., 1999), for example, when a plan completes successfully a success method is invoked, or a failure method if the plan fails. We use these methods to recognize when a plan completes. A time-out mechanism is used to detect when a plan does not complete.

\subsection{Testing Plan Cycles}

In Section 4 we show the order in which the plans should be tested due to the dependencies in the plan structure. For example, in Figure 2 the success of plan $P 0$ depends on the success of either plan $P 2$ or plan $P 3$. These dependencies may on some occasions be cyclic. For example, there is a cyclic dependency between plans $P 0, P 2$ and $P 1$. In this special case we cannot test each plan individually as they are dependent on each other. Hence, such plans are considered as a single unit which we shall term cyclic plans.

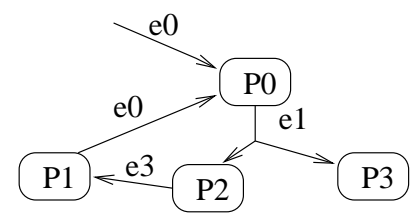

Figure 2: Plan Dependencies

Each plan in the cycle is tested for the aspects discussed above, and in addition the following aspects are tested with respect to the cycle that they form:

- Does the cycle exist at run-time? If the cycle never occurs at run-time then the developer of the system should be notified, as the cycle may have been a deliberate design decision. ${ }^{5}$

- Does the cycle terminate? Using a pre-defined maximum limit for the number of iterations in the cycle, we can determine if the cycle exceeds that limit and warn the user if it does.

\footnotetext{
${ }^{4}$ When deployed, a plan may well fail due to some change in the environment after the time it was selected. However, in the controlled testing situation where there are no external changes, then a plan that does not complete properly (due to failure at some step) should not have been selected.

${ }^{5}$ Alternatively the cycle can be detected at design time and the developer asked whether it is expected to occur at runtime. This information can then be used in testing.
}

\subsection{Testing Events}

An event as we generalized previously is either a percept, a message, or an event within the agent. The purpose of the event is to trigger the activation of a plan. Each event unit is tested for the following:

- Is the event handled by some plan? If the event does not trigger a plan, it could be due to two reasons. The first is if there is no plan that handles that particular event (which is easily checked by the compiler). The second is if the context conditions of all the plans that handle the event are false. This is a test for coverage.

- Is there more than one plan applicable for the event? If at design time the developer has indicated that only one plan is expected to be applicable, then the existence of multiple applicable plans for a given situation (referred to as overlap) is an error.

Mistakes in specification of context conditions in plans, leading to unexpected lack of coverage, or unexpected overlap, are common causes of error in agent programming. Consequently it is a good idea to warn the user if this occurs (though they can also specify that it is expected in which case no warning need be generated).

\section{Testing Process : Overview}

The unit testing process consists of the following steps:

1. Determination of the order in which the units are to be tested.

2. Development of test cases with suitable input value combinations.

3. Augmentation of the code of the system under test with special testing code to facilitate the testing process.

4. Testing, gathering of results, analysis and generation of an appropriate test report.

All of the above steps are automated and can be performed on a partial implementation of the system if needed. This supports the test as you go approach of unit testing.

In this section we briefly discuss the process of testing each type of unit. In the sections to follow we present the method for determining the order in which the units are to be tested and a mechanism for generating practically feasible input combinations for the test cases for each unit. Due to space limitation we do not discuss the implementation of augmenting the code of the system under test or the process of the report generation. 


\subsection{The Testing Framework}

Figure 3 shows an abstract view of the testing framework for a plan unit. It has two distinct components, the test-driver and the subsystem under test. The testdriver component contains the test-agent, testing specific message-events that are sent to and from the test-agent, and a plan (test-driver plan) that initiates the testing process. This plan is embedded into the subsystem under test as part of the code augmenting process. The subsystem under test is the portion of the system that is needed for testing of the relevant unit and includes the necessary data and beliefsets, the supporting hierarchy of the key plans and the key units. The supporting hierarchy of a key plan is the events and plans on which it is dependent for full execution. For testing a plan, the key units are the plan itself, and its triggering event. For testing an event the key units are the event and all plans for which that event is a trigger. For testing a plan cycle the key units are all plans in the cycle and their triggering events.

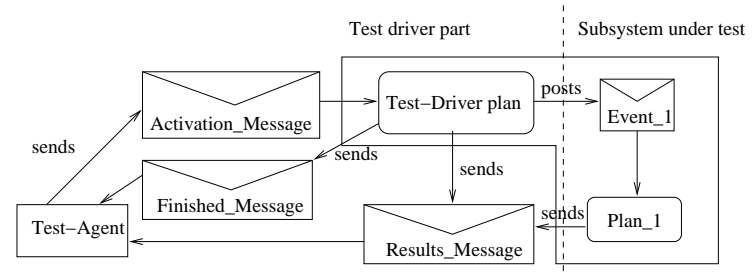

Figure 3: Abstract Testing Framework for a Plan

Figure 3 illustrates the steps in the testing process: the test-agent generates the test cases, and runs each test case by sending an activation message to the testdriver plan; the test-driver plan sets up the input and activates the subsystem under test that executes and sends information (via specially inserted code) back to the test-agent; when testing is complete the testagent generates a report which addresses the questions we have discussed related to each unit in section 2.

Plans that form a cyclic dependency (a cyclic plan set) need to be tested together. In addition to testing each plan in the set, in the same way as a single plan, the specific questions about the cyclic dependency need to be assessed. Does the cycle occur at runtime? Does the cycle terminate?

\section{The Order of Testing}

Recall that an event may be handled by one or more plans and each plan may post sub-tasks. The success of the top level plans is partly dependent on the success of the plans triggered by the sub-tasks (if

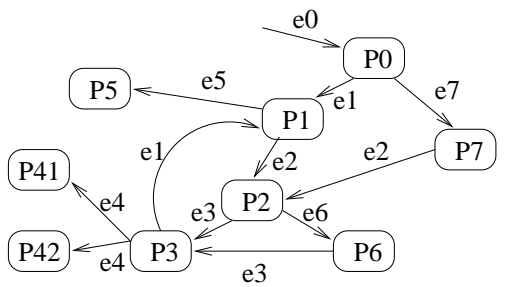

Figure 4: Testing order

any). The order of testing is, therefore, bottom-up where we test a unit before we test any other unit that depends on it. For example, from Figure 4 we test Plan $P 7$ before we test plan $P 0$. The complicating factor is the presence of cyclic dependencies. Plans that form cyclic dependencies are to be tested together as a single unit as previously described.

In order to determine the order of testing we apply the following steps. We use Figure 4 as an example design and abbreviate the following: Plan Test - PT; Event Test - ET; Cyclic Plans Test - CT.

1. We perform a modified depth-first search outlined in Figure 5, which performs a typical depthfirst search but also identifies cyclic dependencies as well as plans that share the same trigger event (for testing coverage and overlap). The order returned by this algorithm for our example is: $\mathrm{PT}(\mathrm{P} 5), \mathrm{CT}(\mathrm{P} 3, \mathrm{P} 1, \mathrm{P} 2), \mathrm{PT}(\mathrm{P} 41), \mathrm{PT}(\mathrm{P} 42)$, ET(e4), PT(P3), CT(P6, P3, P1, P2), PT(P6), PT(P2), PT(P1), PT(P7), PT(P0).

2. From the above order, we can eliminate all unit test of plans that are part of any cyclic dependency as they will be tested when the cyclic plans are tested. The resulting ordered set is: PT(P5), CT(P3，P1，P2)，PT(P41)，PT(P42), ET(e4), CT(P6, P3, P1, P2), PT(P7), PT(P0).

3. In the order above the cyclic plans are not in the correct order as they must be tested only when all of its plans' children have been tested. For instance P41 is a child of P3. This re-ordering is a trivial operation and when complete reveals: PT(P5), PT(P41), PT(P42), ET(e4), CT(P3, P1, P2), CT(P6, P3, P1, P2), PT(P7), PT(P0).

4. The final step is to combine cyclic dependencies that overlap. By overlap we mean cycles that have at least one plan in common. In our example one cycle is a sub-set of the other hence when merged the resulting final order of testing is: $\mathrm{PT}(\mathrm{P} 5)$, PT(P41), PT(P42), ET(e4), CT(P6, P3, P1, P2), PT(P7), PT(P0). 


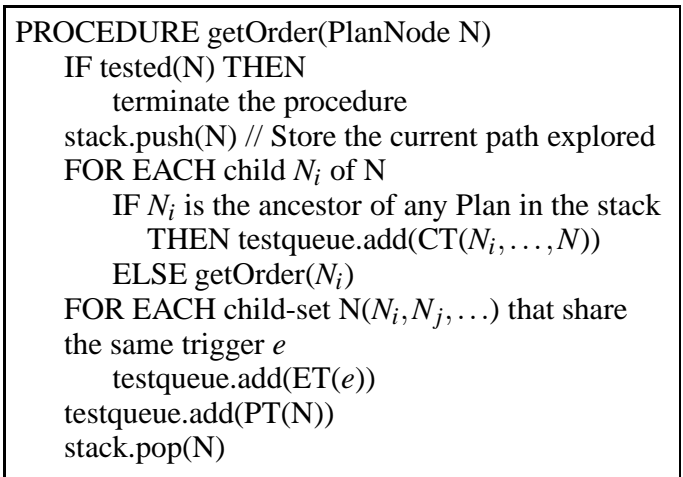

Figure 5: Testing order : Algorithm

\section{Test Case Input Generation}

The variables that we consider as test parameters are those within the context conditions or body ${ }^{6}$ of the plans to be tested and the variables of the entryevent. The entry-event is the initial trigger event when testing a plan, or is the event itself when testing an event. The variables within the event may be used within the plan body. We need to generate practical combinations of these variables to adequately test the plans and events.

There are 3 steps in generating value combinations that form different test cases:

1. Variable extraction from the design documents.

2. Generation of Equivalence Classes, which is a heuristic for reducing the size of the input range. While the concept of Equivalence Classes is not novel, we have adopted our own techniques in applying the heuristic.

3. Generating the input combinations from the equivalence classes using a heuristic to reduce the number of different combinations.

\subsection{Extraction of variables}

The detailed description of plans and events in our design documents contains a list of variables and their types. For variables in context conditions we also have a list of conditions that must be satisfied for the context condition to return True. We call values that satisfy these conditions valid variables for the context condition. Following are some examples of such variables and their associated conditions.

stock, int, $\geq 0$; $\leq 200$;

price, float, $>0.0$;

bookName, string, !=null;

\footnotetext{
${ }^{6}$ We have not yet implemented the use of variables in the body other than those in the context condition and the event. However the information is available from the design and follows the same principles.
}

$$
\begin{gathered}
\text { supplier, SupplierType, }==\text { "Amazon", } \\
==\text { "Powells"; }
\end{gathered}
$$

In our testing framework we define four basic variable types: integer, float, string and enumerated. Other types are considered as special cases of these four basic ones. For example, boolean is considered as a special case of enumerated, and double is a special case of float. The definition of the enumerated types must be contained within the design. For example, the enumerated type SupplierType may be defined as:

[EnumType, SupplierType, \{“Amazon”,

"Angus\&Robertson", "Powells", "Dymocks"\}].

\subsection{The generation of ECs}

It is not possible to create test cases for every valid value of a variable since some domains are infinite, such as $(0.0,+\infty)$ Additionally we wish to test with some invalid values. Even for non-infinite domains the number of test values may be extremely large. To address this issue we use the approach of equivalence partitioning (Patton, 2005, p.67) to obtain a set of representative values. An Equivalence Class (EC) (Binder, 1999, p.401) is a set of input values such that if any value is processed correctly (or incorrectly), then it is assumed that all other values will be processed correctly (or incorrectly). We consider the open intervals and the boundary values of the variable domains to generate ECs, as the former gives equivalent valid values and the latter are edge values that should be checked carefully during testing. We also consider some invalid values.

An EC that we define has five fields:

1. var-name: The name of the variable.

2. Index: A unique identifier.

3. domain: An open interval or a concrete value.

4. validity: Whether the domain is valid or invalid.

5. sample: A sample value from the domain: if the domain is an open interval (e.g. $(0.0,+\infty))$, it is a random value of this interval (e.g 778); if the domain is a concrete value $(x=3)$, it is this value.

Table 1 gives the equivalence classes for the example variables above.

When generating the ECs for a particular variable we use the following rules (we refer to Table 1):

- One EC is generated for each boundary value of the variable. The sample value of that EC is the boundary value. E.g., for variable 'stock', EC-2 and EC-4 are created using the boundary values.

- For an integer or float variable, one EC is generated for each open interval between two neighbouring boundary values. The sample value is a random value in this interval. E.g., for variable 'stock', EC-1 EC-3, and EC-5 are generated using boundary value intervals. 


\begin{tabular}{|c|c|c|c|c|}
\hline variable & index & domain & valid & sample \\
\hline \multirow{3}{*}{ stock } & EC-1 & $(-\infty, 0)$ & no & -823 \\
\cline { 2 - 5 } & EC-2 & 0 & yes & 0 \\
\cline { 2 - 5 } & EC-3 & $(0.0,200)$ & yes & 139 \\
\cline { 2 - 5 } & EC-4 & 200 & yes & 200 \\
\cline { 2 - 5 } & EC-5 & $(200,+\infty)$ & no & 778 \\
\hline \multirow{3}{*}{ price } & EC-1 & $(-\infty, 0.0)$ & no & -341.0 \\
\cline { 2 - 5 } & EC-2 & 0.0 & yes & 0.0 \\
\cline { 2 - 5 } & EC-3 & $(0.0,+\infty)$ & yes & 205.0 \\
\hline book & EC-1 & NULL & no & NULL \\
\cline { 2 - 5 } supplier & EC-2 & not NULL & yes & "random" \\
\cline { 2 - 5 } & EC-1 & "Amazon" & yes & "Amazon" \\
\cline { 2 - 5 } & & "Angus\& & yes & "Angus\& \\
\cline { 2 - 5 } & EC-3 & "Pobertson" & & Robertson" \\
\cline { 2 - 5 } & EC-4 & "Dymocks" & yes & "Powells" \\
\hline \multirow{2}{*}{} & & "Dymocks" \\
\hline
\end{tabular}

Table 1: ECs of all variables

- For a string variable, one EC is generated to represent the domain of valid values. The sample value is a random string that is not a valid value. E.g., for variable 'bookName' EC-2 is such an EC.

- For an string variable, one EC is generated to accommodate the NULL value.

- For an enumerated variable, one EC is generated for each value of the enumerated type.

The generated ECs for the sample variables given above are displayed in Table 1.

\subsection{Reducing the size of the test set}

It is straightforward to generate the set of all possible combinations of variable ECs, which could then be used for the value combinations for test cases. However the number of the combinations may still be quite large. In our example, there are 120 combinations of all the variables. This number can be reduced further by using the approach of combinatorial design (Cohen et al., 1997). This approach generates a new set of value combinations that cover all $n$-wise $(n \geq 2)$ interactions among the test parameters and their values in order to reduce the size of the input data set. Hartman and Raskin have developed a software library called CTS (Combinational Testing Service) ${ }^{7}$ which implements this approach. We do not expand on these techniques as we do not modify them by any means. Using this software we are able to reduce the set of 120 combinations to a smaller set of 24 combinations. We then use the sample value from each EC to obtain the concrete test data for each test case that will be run. Table 2 shows some sample value combinations from the reduced list, where each combination represents the input to a unique test case. Whether or not this method is used can be determined depending on the

\footnotetext{
${ }^{7}$ http: //www. alphaworks.ibm. com/tech/cts
}

\begin{tabular}{|c|c|c|c|c|}
\hline index & stock & price & bookName & supplier \\
\hline 1 & 139 & 205.0 & "random" & "Amazon" \\
\hline 2 & 200 & 205.0 & "random" & "Amazon" \\
\hline$\ldots$ & $\ldots$ & $\ldots$ & $\ldots$ & $\ldots$ \\
\hline 23 & 139 & 205.0 & NULL & "Powells" \\
\hline 24 & 200 & 205.0 & "random" & "Powells" \\
\hline
\end{tabular}

Table 2: List of EC value combinations

number of total combinations. It is also possible to differentiate between valid and invalid data, reducing the number of combinations for invalid data, while using all possibilities for valid data to ensure that all options through the code are exercised.

\section{Case Study}

As a first step in evaluating our testing framework we took a sample agent system, systematically introduced all types of faults discussed in section 2 into the system and used it as input to the testing framework. The testing framework successfully uncovered each of these faults in the automated testing process.

The sample system that we used, was the Electronic Bookstore system as described in (Padgham and Winikoff, 2004). This is an agent-based system dealing with online book trading, containing agents such as Customer Relations, Delivery Manager, Sales Assistant and Stock Manager agents. We used the Stock Manager agent as the agent under test (AUT), and specifically edited the code to introduce all identified types of faults. The testing framework generator automatically generated the testing framework for the testable units of the Stock Manager agent, and then executed the testing process for each unit following the sequence determined by the testing-order algorithm. For each unit, the testing framework ran one test suite, which was composed of a set of test cases, with each case having as input one of the value combinations determined.

For example, as discussed earlier, one kind of fault that can occur is that a particular subtask is never posted from a plan, despite the fact that the design indicates it should be. In the Stock Manager the plan Out of stock response had code that, when the book is needed urgently and the number of ordered books is less than 100, checks if the default supplier currently has stock and if not posts the subtask Decide supplier. We modified the body of the code for Out of stock response so that a condition check would always be false, thus leading to the situation that the Decide supplier subtask event would in fact never be posted.

The plan Out of stock response had as its trigger event No stock which included the boolean variable Urgent. The context condition of this plan was: 


\begin{tabular}{|c|c|c|c|c|}
\hline variable & index & domain & valid & sample \\
\hline \multirow{3}{*}{ BookID } & EC-1 & $(0,+\infty)$ & yes & 11 \\
\cline { 2 - 5 } & EC-2 & $(-\infty, 0)$ & no & -2 \\
\cline { 2 - 5 } & EC-3 & 0 & yes & 0 \\
\hline \multirow{3}{*}{$\begin{array}{c}\text { Number } \\
\text { Ordered }\end{array}$} & EC-1 & $(0,+\infty)$ & yes & 8 \\
\cline { 2 - 5 } & EC-2 & $(-\infty, 0)$ & no & -9 \\
\cline { 2 - 5 } & EC-3 & 0 & no & 0 \\
\hline \multirow{2}{*}{ Urgent } & EC-1 & yes & yes & yes \\
\cline { 2 - 5 } & EC-2 & no & yes & no \\
\hline
\end{tabular}

Table 3: ECs of all variables

(BookID $\geq 0$ AND NumberOrdered $>0$ ). Within the body of the plan we had the code :

$$
\begin{array}{ll}
\text { IF } & \text { Urgent=YES AND NumberOrdered }<100 \\
\text { THEN } & \text { postEvent }(\text { Decide supplier }) \\
\text { ENDIF } &
\end{array}
$$

To introduce the fault into the system we modified the IF condition above to be :

IF Urgent=YES AND NumberOrdered $<0 \quad$ which will of course result in Decide supplier never being posted. The input arguments for the test are then BookID, NumberOrdered and Urgent, with the following specifications:

BookID, int, $\geq 0$

NumberOrdered, int, $>0$

Urgent, boolean

This gives the equivalence classes as shown in table 3 , giving 18 possible combinations of values shown in table 4, which is reduced to 9 if the combinatorial testing reduction is used.

This error was discovered by the testing system by analysing the results of the test suite and observing that Decide supplier was never posted. The following is an example of the warning message that is supplied to the user:

Type of Fault: Subtask never posted WARNING: The event Decide supplier is never posted in any test case. Value combinations used in test suite were: ${ }^{8}$

BookID $=11 \quad$ NumberOrdered $=8 \quad$ Urgent=yes

BookID $=0 \quad$ NumberOrdered $=8 \quad$ Urgent $=$ yes

BookID $=11 \quad$ NumberOrdered $=8 \quad$ Urgent $=$ no

BookID $=0 \quad$ NumberOrdered $=8 \quad$ Urgent $=$ no

If some other value combination would result in posting of event Decide supplier, please provide these values to the testing system.

\section{Discussion}

The need for software testing is well known and accepted. While there are many software test-

\footnotetext{
${ }^{8}$ Only valid values are provided as invalid values would

\begin{tabular}{|c|c|c|c|c|}
\hline index & BookID & $\begin{array}{l}\text { Number } \\
\text { Ordered }\end{array}$ & Urgent & Validity \\
\hline 1 & EC-1 (11) & $\overline{E C-1(8)}$ & EC-1 (yes) & \multirow[t]{4}{*}{ valid } \\
\hline 2 & EC-3 (0) & $\overline{E C-1(8)}$ & EC-1 (yes) & \\
\hline 3 & EC-1 (11) & EC-1 (8) & EC-2 (no) & \\
\hline 4 & EC-3 (0) & EC-1 (8) & EC-2 (no) & \\
\hline 5 & EC-1 (11) & EC-2 (-9) & EC-1 (yes) & \multirow[t]{14}{*}{ invalid } \\
\hline 6 & EC-1 (11) & EC-2 (-9) & EC-2 (no) & \\
\hline 7 & EC-1 (11) & EC-3 (0) & EC-1 (yes) & \\
\hline 8 & EC-1 (11) & EC-3 (0) & EC-2 (no) & \\
\hline 9 & EC-2 (-2) & EC-1 (8) & EC-1 (yes) & \\
\hline$\overline{10}$ & $\overline{E C}-2(-2)$ & $\overline{E C-1(8)}$ & EC-2 (no) & \\
\hline 11 & $\mathrm{EC}-2(-2)$ & $\mathrm{EC}-2(-9)$ & EC-1 (yes) & \\
\hline 12 & EC-2 (-2) & EC-2 (-9) & EC-2 (no) & \\
\hline 13 & $\overline{E C-2(-2)}$ & $\mathrm{EC}-3(0)$ & EC-1 (yes) & \\
\hline 14 & EC-2 (-2) & EC-3 (0) & EC-2 (no) & \\
\hline 15 & EC-3 (0) & $\overline{\mathrm{EC}-2(-9)}$ & $\overline{\mathrm{EC}-1 \text { (yes) }}$ & \\
\hline 16 & EC-3 (0) & $\overline{E C-2(-9)}$ & EC-2 (no) & \\
\hline 17 & EC-3 (0) & EC-3 (0) & $\overline{\text { EC-1 (yes) }}$ & \\
\hline 18 & $\overline{E C-3(0)}$ & $\overline{E C-3(0)}$ & EC-2 (no) & \\
\hline
\end{tabular}
not cause the plan to run and are hence irrelevant for this error.
}

Table 4: List of Equivalence Class combinations

ing frameworks for traditional systems like ObjectOriented software systems, there is little work on testing Agent-Oriented systems. In particular to the best of our knowledge there is no testing framework that is integrated into the development methodology.

In this paper we present part of a framework for testing agent systems that we have developed, which performs model based unit testing. We have identified as units, plans, events that are handled by multiple plans, and plans that form cyclic dependencies. We have presented an overview of the testing process and mechanisms for identifying the order in which the units are to be tested and for generating the input that forms test cases.

There has been some work on testing agent based systems in recent years (e.g (Zheng and Alagar, 2005; Seo et al., 2004)). The former provides an approach to compare the properties of the agent and the observable behaviours with the specification of the system, by building a behavioral model for the system using extended state machines. The latter studied how to build a state-based model for an agent-based system using extended Statecharts, and then proposed an approach to generate test sequences. Both of the above work is based on conformance testing, which tests if the system meets the business requirements and are restricted to black-box testing. In contrast to these approaches, our work looks at fault directed testing which tests the internal processes of the system and not the business requirements. Our approach is also integrated with the design methodology and supports testing at early stages of development.

There are other work on multi-agent testing that defines agents as test units (e.g (Caire et al., 2004; 
Rouff, 2002)). We however, explore the internals of an agent and choose plans and events as test units.

While we obtain and use more structural information than standard black box testing, we are limited in the information we use as we obtain this information from the design. Hence, implementation specific structure is not considered. The testing framework is also reliant on the implementation following the design specification.

Although we have completed the implementation of the testing framework using JACK Intelligent Systems (Busetta et al., 1999), and done some preliminary evaluation as discussed in the previous section, further evaluation is required. For this purpose we intend to use programs developed by post-graduate students as part of an agent programming course.

In this work we have only addressed unit testing, in future work we will extend this work to include integration testing. To this end, we expect to build on existing work (e.g. (Padgham et al., 2005; Coelho et al., 2006)). The former described a debugger which, similar to this work, used design artefacts of the Prometheus methodology to provide debugging information at run-time. Their approach of converting protocol specifications to petri-net representations is of particular relevance to our future work on integration testing. The latter presented a unit testing approach for multi-agent systems based on the use of Mock-Agents, where each Mock-Agent tests a single role of an agent under various scenarios.

As future work we also look to embed the testing functionality into the Prometheus Design Tool (PDT) (Thangarajah et al., 2005). PDT is a tool for developing agent systems following the Prometheus methodology, and includes automated code generation which we hope to extend to generate testing specific code.

\section{Acknowledgements}

We would like to acknowledge the support of the Australian Research Council and Agent Oriented Software, under grant LP0453486.

\section{REFERENCES}

Apfelbaum, L. and Doyle, J. (1997). Model Based Testing. In the 10th International Software Quality Week Conference, CA, USA.

Binder, R. V. (1999). Testing Object-Oriented Systems: Models, Patterns, and Tools. Addison-Wesley Longman Publishing Co., Inc., Boston, MA, USA.

Bresciani, P., Perini, A., Giorgini, P., Giunchiglia, F., and Mylopoulos, J. (2004). Tropos: An Agent-Oriented
Software Development Methodology. Autonomous Agents and Multi-Agent Systems, 8(3):203-236.

Busetta, P., Rönnquist, R., Hodgson, A., and Lucas, A. (1999). JACK Intelligent Agents - Components for Intelligent Agents in Java. Technical report, Agent Oriented Software Pty. Ltd, Melbourne, Australia.

Caire, G., Cossentino, M., Negri, A., Poggi, A., and Turci, P. (2004). Multi-Agent Systems Implementation and Testing. In the Fourth International Symposium: From Agent Theory to Agent Implementation, Vienna.

Coelho, R., Kulesza, U., von Staa, A., and Lucena, C. (2006). Unit Testing in Multi-Agent Systems using Mock Agents and Aspects. In Proceedings of the 2006 International Workshop on Software Engineering for Large-Scale Multi-Agent Systems, pages 83-90.

Cohen, D. M., Dalal, S. R., Fredman, M. L., and Patton, G. C. (1997). The AETG system: An Approach to Testing Based on Combinatiorial Design. Software Engineering, 23(7):437-444.

Deloach, S. A. (2001). Analysis and Design using MaSE and agentTool. In the 12th Midwest Artificial Intelligence and Cognitive Science Conference (MAICS 2001), Miami University, Oxford, Ohio.

El-Far, I. K. and Whittaker, J. A. (2001). Encyclopedia of Software Engineering, chapter Model-Based Software Testing, pages 825-837. Wiley.

Padgham, L. and Winikoff, M. (2004). Developing Intelligent Agent Systems: A practical guide. Wiley Series in Agent Technology. John Wiley and Sons.

Padgham, L., Winikoff, M., and Poutakidis, D. (2005). Adding Debugging Support to the Prometheus Methodology. Engineering Applications of Artificial Intelligence, special issue on Agent-Oriented Software Development, 18(2):173-190.

Patton, R. (2005). Software Testing (Second Edition). Sams, Indianapolis, IN, USA.

Rao, A. S. and Georgeff, M. P. (1995). BDI Agents: From Theory to Practice. In Lesser, V., editor, the First International Conference on Multi-Agent Systems, pages 312-319, San Francisco.

Rouff, C. (2002). A Test Agent for Testing Agents and their Communities. Aerospace Conference Proceedings, 2002. IEEE, 5:2638.

Seo, H.-S., Araragi, T., and Kwon, Y. R. (2004). Modeling and Testing Agent Systems Based on Statecharts. volume 3236, pages $308-321$.

Thangarajah, J., Padgham, L., and Winikoff, M. (2005). Prometheus design tool. In the 4th International Joint Conference on Autonomous Agents and Multi-Agent Systems, pages 127-128, Utrecht, The Netherlands.

Zheng, M. and Alagar, V. S. (2005). Conformance Testing of BDI Properties in Agent-based Software Systems. In APSEC '05: Proceedings of the 12th Asia-Pacific Software Engineering Conference (APSEC'05), pages 457-464, Washington. IEEE Computer Society. 